\title{
Simplified 3-D FE model of thermal conditions inside a shoe
}

\author{
H. Raval ${ }^{1}$, Z. W. Guan ${ }^{2}$, M. Bailey ${ }^{1}$ \& D. G. Covill ${ }^{1}$ \\ ${ }^{I}$ Micro Climate Research Unit, University of Brighton, Brighton, UK \\ ${ }^{2}$ Department of Engineering, University of Liverpool, Liverpool, UK
}

\begin{abstract}
To design thermally comfortable shoes, the knowledge of thermal conditions inside the shoes and the variables affecting those conditions is necessary. A simplified 3-D thermal numerical model of a shoe has been developed. A new approach was adopted to construct the mesh. The model was developed to consider the dry heat transfer in the shoe and convective heat loss from the outer surface of the shoe. The foot was the source of the heat in the model. Model's predictions were compared with the results obtained during the experiments. The predicted in-shoe temperatures correlated reasonably well with the measurements although they were higher than the measurements in some cases. Probable reasons behind some inconsistency between predictions and the measured temperatures have been discussed. The paper concludes that the model's predictions can be improved by incorporating the effect of other variables. Keywords: shoe climate, thermal comfort, thermal model of a shoe.
\end{abstract}

\section{Purpose}

It is important for the body to maintain its core temperature around $37^{\circ} \mathrm{C}$ (Bazett [1]). Depending on the environmental conditions and level of physical activity, the thermoregulatory system of the body controls the blood flow and hence heat being supplied to the skin and the periphery of the body to maintain the core temperature (Bazett [1]). Normally, the released heat from foot's surface can vary from $3 \mathrm{~W}$ to $30 \mathrm{~W}$ depending on the activity level at the particular time (Oakley [2]). The body also controls the generation of sweat and the resulting wet heat transfer. Both types of responses can have a significant effect on the heat loss from the body because of large surface area of the feet. If sweat is not 
allowed to evaporate, it can become uncomfortable and affect the heat transfer by increasing thermal conductivities of the skin, sock and shoe even during the situations where minimal heat loss is desired. Therefore, the material properties of the sock and the shoe are important to enable the body to achieve the intended heat loss from the feet and to remove sweat from the feet quickly.

It is vital to understand the process of heat transfer taking place from the foot to the environment via the sock, in-shoe air and shoe in order to design thermally comfortable shoes. Experiments to understand shoe climate are useful in obtaining in-shoe thermal conditions and correlating these conditions with the perceptions of the subjects. However, the number of experimental conditions which can be evaluated may be limited due to the time and resources involved. Computer models, once validated, could be used to numerically study the effects of different materials, ambient conditions, heat flux and changes in design shoe climate.

\section{Methodology}

\subsection{Experimental measures}

Static experiments were conducted at ambient temperatures of $20^{\circ} \mathrm{C}$ and $32^{\circ} \mathrm{C}$ with 50\% Relative humidity (RH) with nine subjects for each ambient temperature. Prior to the experiments, the subjects bathed their feet in water warmed at $33^{\circ} \mathrm{C}$ to minimise differences in skin temperatures prior to test. The shoes were conditioned in the environmental chamber for one hour prior to the experiments to attain similar temperatures as that of the environment. Sensors were implanted in the upper shoe that recorded the temperatures of the in-shoe air close to the inner boundary of the shoe. Experiments were carried out for 35 minutes with in-shoe temperatures being measured at every 30 seconds.

\subsection{Modelling}

A plaster cast of a foot was taken from a volunteer, who normally wore a UK size nine shoe. The plaster cast was then positioned inside a UK size nine shoe, so the shoe adopted the shape it would in wear to stabilise the shoe to allow it to be cut into coronal plane sections. The plaster cast and the shoe were cut using a hacksaw at approximately $25 \mathrm{~mm}$ intervals apart as shown in the Figure 1.

The sections were used to create a series of 2-D planes by digitising coordinates onto graph paper with key points plotted on the circumference of the inner and the outer boundaries of the shoe and on the boundary of the foot to generate 2-D plots. Geometric conditions of all slices were implemented in a finite element programme through ABAQUS (ABAQUS Inc, Providence, Rhode Island) to create the geometric mesh. The coronal planes provided the outline geometry of the outer and inner shoe, foot and in-shoe air at regular intervals. Subsequent layers between the planes were generated at $6.25 \mathrm{~mm}$ intervals along the coronal axis using eight-node brick elements. The ends of the foot and the shoe had been slightly simplified to minimise the geometrical difficulties although the inner shape of the shoe was undisturbed. 


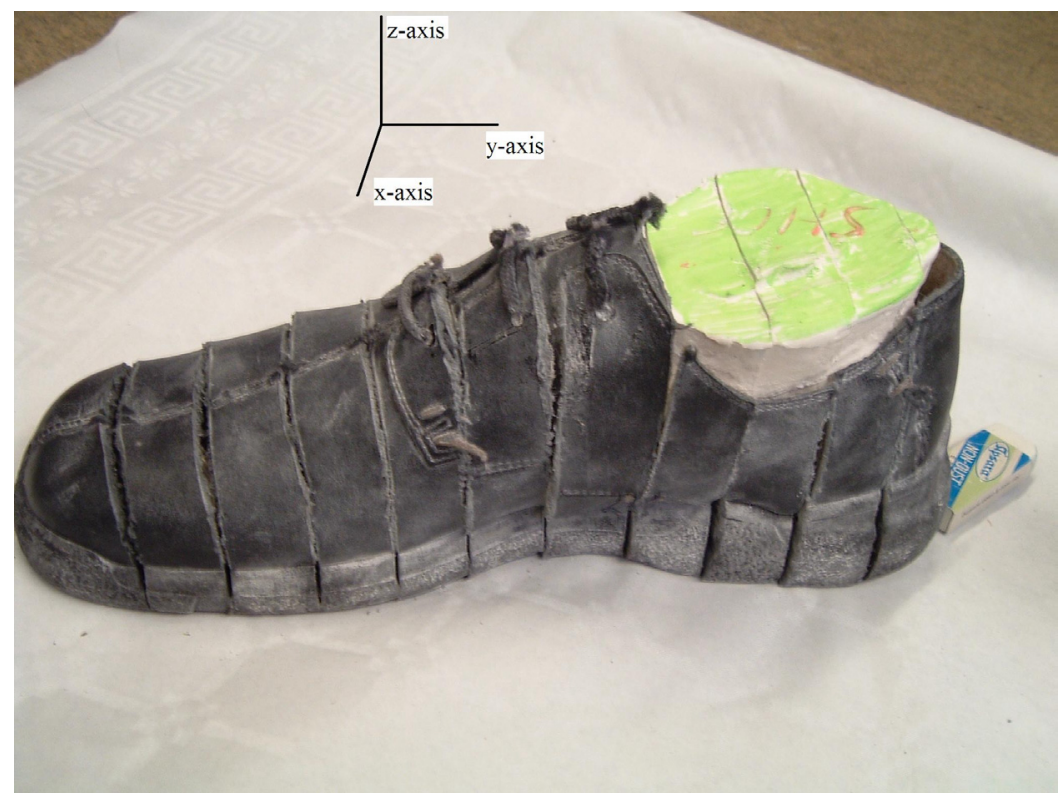

Figure 1: A shoe with plaster cast of a foot cut into slices in at $25 \mathrm{~mm}$ interval.

To represent the in-shoe air, 8-node linear brick and 6-node linear triangular prism elements were generated between the meshed foot and shoe. The air was meshed by filling the gap between corresponding nodes of the foot and shoe in different sections. Some air-pockets were left un-meshed because of the unavailability of eight or six distinct free nodes to create an element. These were considered to encompass a negligibly small volume.

Figure 2 shows 3-D mesh of a shoe as it would be expected to appear in wear, the foot and the in-shoe air. The ends of the shoe and the foot were simplified to overcome the geometrical difficulties faced by ABAQUS. However, the boundary of the inner shoe was undisturbed by this simplification.

The foot was the source of heat in the model. In this simplified model, the foot released heat to the system uniformly from its surface at a rate of $13 \mathrm{~W}$. Material properties were assigned to the three different materials. The shoe material was to be homogenous and its thermal conductivity to be isotropic. The foot was also assigned uniform material properties. Table 1 shows the material properties used in the model.

The model calculates the dry heat transfer taking place in the shoe due to conduction. It also incorporates heat loss to the environment from the outer surface of the shoe due to convection by defining the film coefficient $(\mathrm{h}=20.0$ $\mathrm{W} / \mathrm{m}^{2} \cdot \mathrm{K}$ ). Surface interactions between different surfaces in contact ware defined to allow the model to calculate the heat conduction taking place from the foot to in-shoe air, from the in-shoe air to shoe and from the foot to shoe in the absence of in-shoe air. 
404 Advanced Computational Methods in Heat Transfer IX

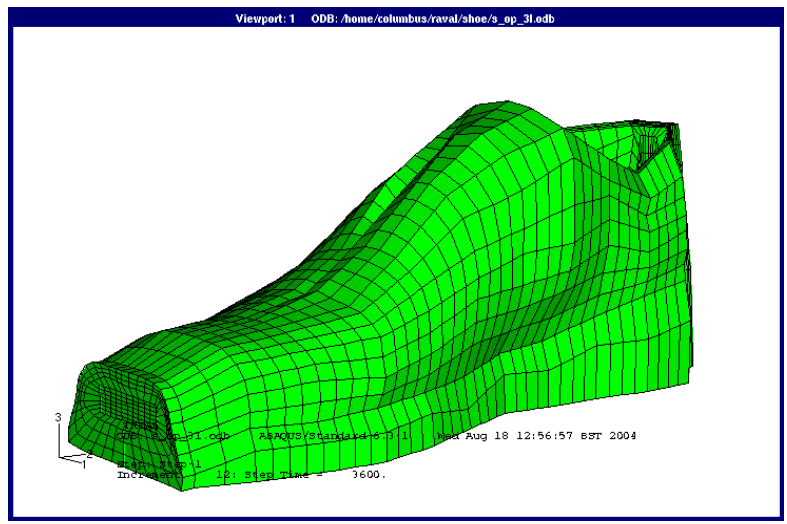

(i) Shoe

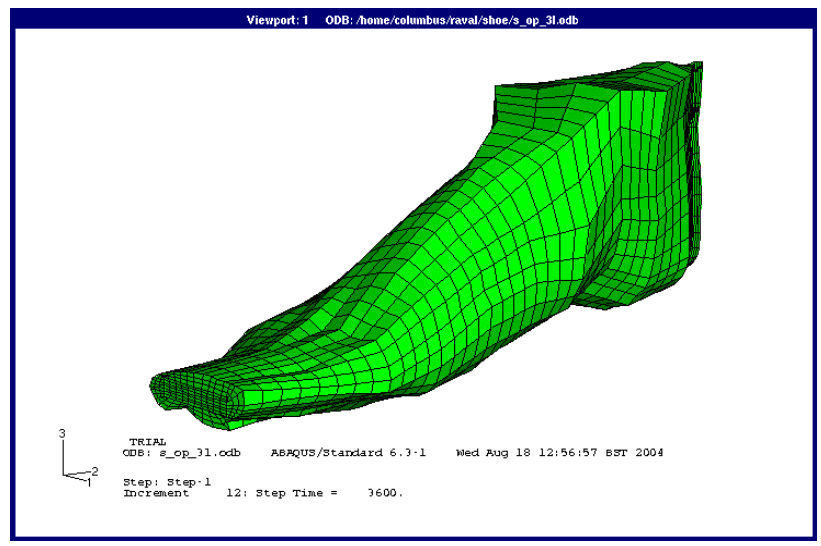

(ii) Foot

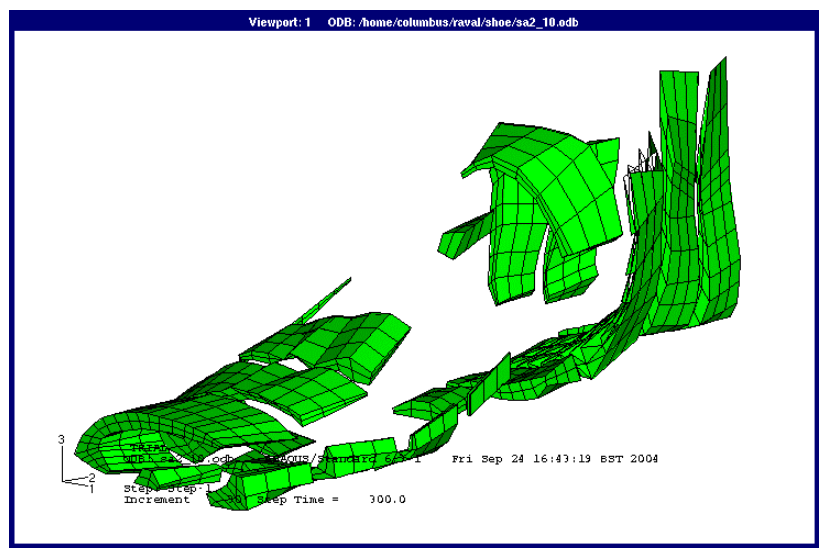

(iii) In-shoe air

Figure 2: $\quad 3-D$ meshes representing a shoe, foot and in-shoe air. 
Table 1: $\quad$ Material properties used in the numerical model.

\begin{tabular}{|l|l|l|l|l|}
\hline Part & $\begin{array}{l}\text { Thermal } \\
\text { Conductivity } \\
(\mathrm{W} / \mathrm{m} \cdot \mathrm{K})\end{array}$ & $\begin{array}{l}\text { Specific } \\
\text { Heat } \\
(\mathrm{J} / \mathrm{Kg} \cdot \mathrm{K})\end{array}$ & $\begin{array}{l}\text { Density } \\
\left(\mathrm{Kg} / \mathrm{m}^{3}\right)\end{array}$ & Source \\
\hline Shoe & 0.16 & 2010 & 998 & Covill [3] \\
\hline Foot & 0.20 & 1260 & 810 & Covill [3] \\
\hline In-shoe air & 0.026 & 1005 & 1.2 & Covill [3] \\
\hline
\end{tabular}

To simulate with the experimental data, the initial temperature of the foot in the model was taken as $33^{\circ} \mathrm{C}$. The model also assumed that the initial temperatures of the shoe, in-shoe air and environment were the same, as those of the environmental chamber where the shoes were conditioned prior to the experiments.

\section{Results}

To validate the model, the predicted temperatures at in-shoe locations were compared with measured in-shoe temperatures. Figure 3 shows the comparison between the predicted and the measured in-shoe temperatures for the toe-region under two different ambient conditions. The temperature for the toe-region was obtained by averaging four different in-shoe temperatures over the front foot.

Figures 4 and 5 show the measured and predicted temperature for the arch and midfoot under two different controlled ambient conditions. Temperatures for these two locations were measured at single locations unlike the toe region.

\section{Discussion}

The predicted results have showed similar trends in changes of temperatures at particular in-shoe locations although the magnitude of these changes, sometimes, was different from the measured results.

For the toe region at $20^{\circ} \mathrm{C}$, the predictions from the model fall within one standard error of the mean temperatures obtained experimentally after 15 minutes. However, in the hot environment of $32^{\circ} \mathrm{C}$, the predicted results are about $6 \%$ lower than those measured. For the arch and midfoot, in Figures 4 and 5 , the predictions generally fall within the standard error of the mean temperature at the environmental temperature of $32^{\circ} \mathrm{C}$. At $20^{\circ} \mathrm{C}$, the predictions overestimate the mean measured temperature by around $7 \%$ and $16 \%$ for the arch and midfoot respectively. 

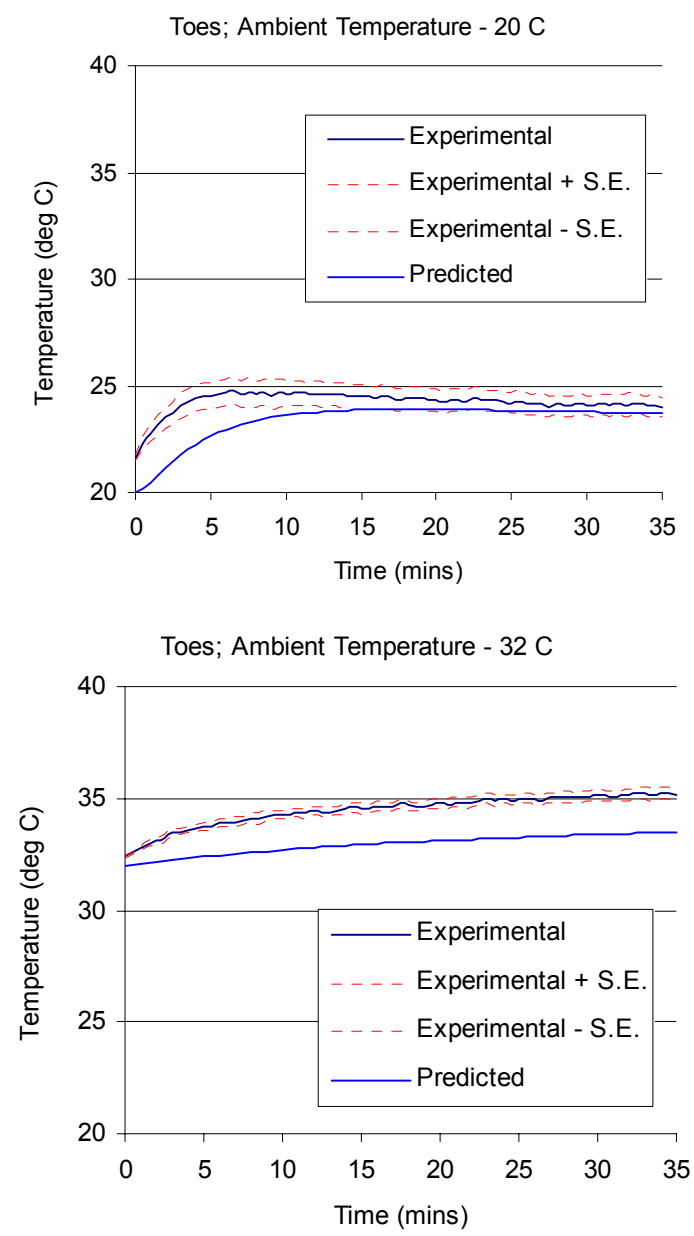

Figure 3: Comparison between the predicted and measured in-shoe temperature for the toe region at ambient temperatures of $20^{\circ} \mathrm{C}$ and $32^{\circ} \mathrm{C}$.

Various factors may have affected the predictions. Although the shoe was conditioned in a controlled environment for an hour at $20^{\circ} \mathrm{C}$, the initial temperature for all three locations was not exactly the same as that of the environment. In the model, the heat flux was constant irrespective of ambient conditions and was applied uniformly over the surface of the foot; however, the heat flux is a function of ambient conditions and the locations on the foot. To apply the heat to the foot accurately, the knowledge of relationship between the environmental conditions and the heat released from the different regions of the 

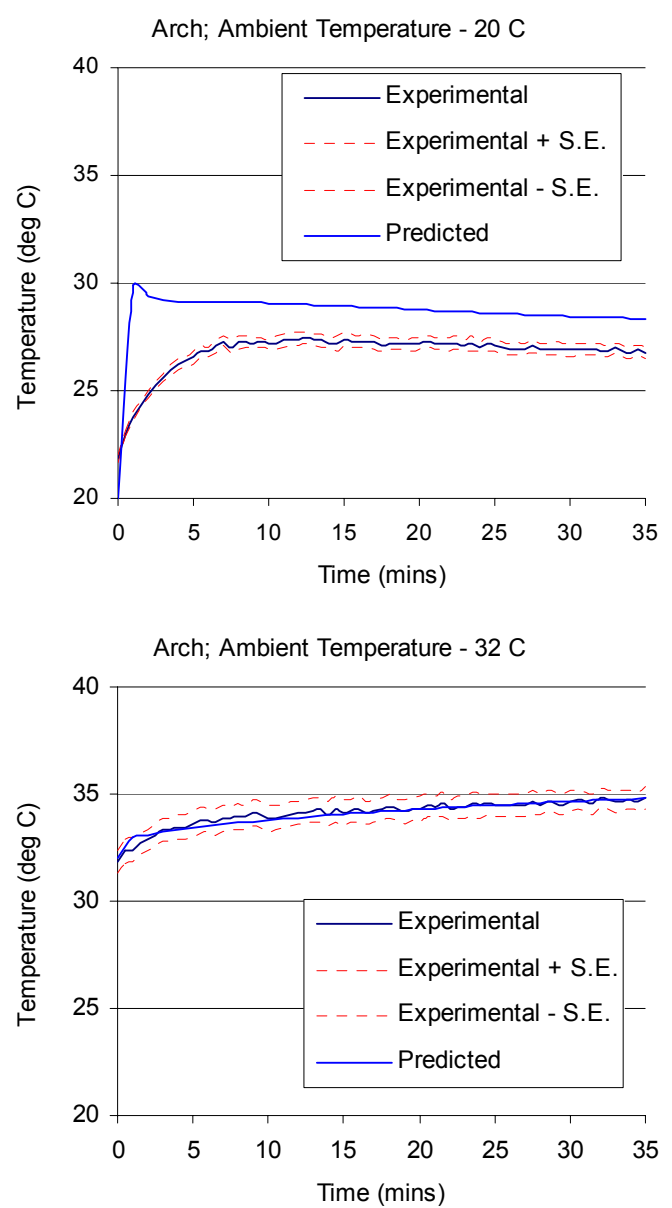

Figure 4: Comparison between the predicted and measured in-shoe temperature for the arch at ambient temperatures of $20^{\circ} \mathrm{C}$ and $32^{\circ} \mathrm{C}$.

foot is essentially required. Since the geometry of the tow-region was simplified and thus the surface area reduced, it could also be one of the reasons for lower heat loss from the toe-region at $20^{\circ} \mathrm{C}$.

This preliminary model does not consider the effect of wet heat transfer on the in-shoe thermal conditions. It may have a noticeable effect on the shoe climate especially at higher ambient temperatures and/or relative humidity. The material properties of the shoe used in experiments may not have been the same as those of the shoe used in the model. 

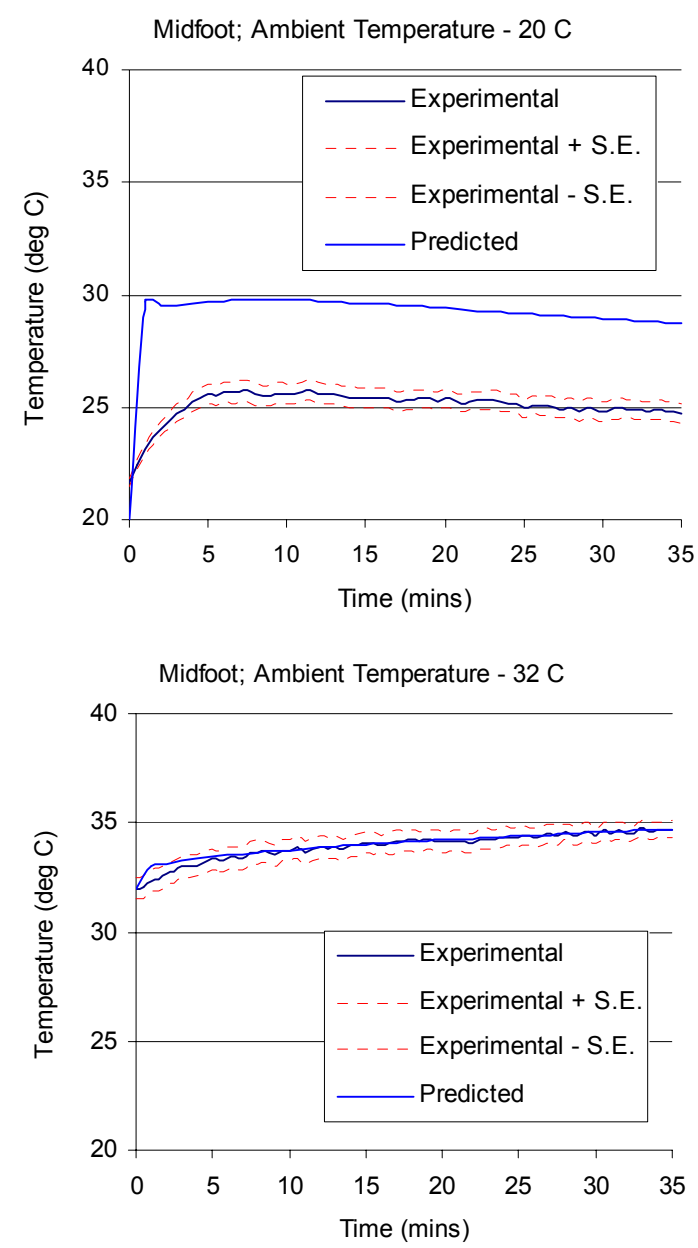

Figure 5: Comparison between the predicted and measured in-shoe temperature for the midfoot at ambient temperatures of $20^{\circ} \mathrm{C}$ and $32^{\circ} \mathrm{C}$.

Although great care was taken while cutting the shoe and plaster cast in the slices, some of the material of the plaster cast was lost during cutting. The deformed shoe also partially regained its earlier un-deformed shape once it was cut into the slices as some tension was removed. Also the absence of the sock in the model means its effect on the rate of heat transfer is ignored.

The model, however, showed promising results by describing the nature of changes taking place in the shoe, including the initial rapid rise in the temperature, with time in static conditions. It also predicted the lowest temperature for the toe-region of all three locations through out 35 minutes of 
experiment at ambient temperature of $20^{\circ} \mathrm{C}$, which was the case during experiments. The model also successfully predicted temperatures for the arch and midfoot at ambient temperature of $32^{\circ} \mathrm{C}$.

\section{Conclusion}

A simplified 3-D thermal model was developed. The mesh was constructed manually using the measurements of the outline of the shoe, foot and in-shoe air. The model incorporated the effect of dry heat transfer in the shoe and convective heat loss from the shoe surface in terms of shoe climate. The model was validated against the corresponding experimental results and reasonable correlation was obtained. The predictions showed a similar pattern to the changes of the in-shoe temperatures with time for some conditions and locations.

Investigation of mesh parameters, heterogeneous material properties and realistic variations in heat flux from the foot could yield better predictions of test results by the model.

\section{Acknowledgement}

The authors would like to thank Clarks International for funding the research and providing the shoes for the experiments.

\section{References}

[1] Bazett H.C., the regulation of body temperatures. Physiology of Heat Regulation and the Science of Clothing, ed. L.H. Newburgh, Hafner Publishing Co.: London, pp. 109-192, 1968.

[2] Oakley E.H.N., the design and function of military footwear: a review following experiences in the South Atlantic. Ergonomics, 27, pp. 631-637, 1984.

[3] Covill D.G., Experimental and numerical modelling of the temperature and moisture distributions in footwear, PhD thesis, University of Brighton, 2005. 